\title{
Um Mapeamento Sistemático da Literatura sobre o uso de Metodologias Ativas durante o Ensino de Programação no Brasil
}

\author{
Ivanilse Calderon $^{1,3}$, Williamson Silva ${ }^{2}$, Eduardo Feitosa $^{1}$ \\ ${ }^{1}$ Instituto de Computação (IComp) - Universidade Federal do Amazonas (UFAM) \\ Manaus, AM - Brasil \\ ${ }^{2}$ Laboratory of Empirical Studies in Software Engineering (LESSE) - Departamento \\ de Engenharia de Software - Universidade Federal do Pampa (UNIPAMPA) \\ - Alegrete, RS - Brasil \\ ${ }^{3}$ Instituto Federal de Educação, Ciência e Tecnologia de Rondônia (IFRO) \\ Campus Porto Velho Zona Norte - Porto Velho, RO - Brasil

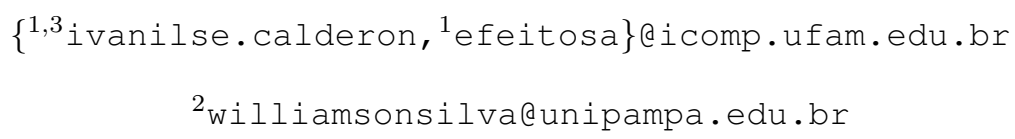

\begin{abstract}
Teaching programming is a challenge for instructors of Computer Science courses. Instructors have been adopting Active Learning Methodologies (ALMs) into teaching practices of computer programming to minimize the challenges faced in the classroom. In this sense, we performed a systematic literature mapping to summarize the main ALMs adopted by instructors during teaching programming in undergraduate courses in the Brazilian context. We identified main ALMs, and Educational Games and Gamification are the most adopted by Brazilian instructors. We also identified students' perceptions about the use of these ALMs in the classroom.
\end{abstract}

Resumo. O ensino de programação é um desafio para os docentes dos cursos de Computação. Como forma de tentar minimizar os desafios enfrentados em sala de aula, os docentes vem adotando as Metodologias Ativas (MAs) em suas práticas de ensino e aprendizagem de programação de computadores. Nesse sentido, conduziu-se um Mapeamento Sistemático da Literatura para sumarizar as principais MAs adotadas pelos docentes durante o ensino de programação nos cursos de graduação no cenário brasileiro. A partir dos resultados, foram identificadas dez tipos de MAs, sendo Jogos Educacionais e Gamificação as mais adotadas pelos docentes brasileiros. Foram identificadas também as percepções dos estudantes sobre o uso destas MAs em sala de aula.

\section{Introdução}

O processo de aprendizagem em disciplinas de programação de computadores, em cursos da área de Computação (conhecidas na literatura como CS1 e CS2), é uma atividade complexa e difícil [Luxton-Reilly et al. 2018]. Estas disciplinas requerem que os estudantes desenvolvam, ao longo da aprendizagem, diferentes habilidades como alta capacidade cognitiva de abstração dos problemas, resolução de problemas, raciocínio e pensamento 
lógico [Raj et al. 2018]. Para que os estudantes desenvolvam tais habilidades, os docentes precisam adotar novas estratégias de ensino e obter uma postura mais transformadora em sala de aula, proporcionando um ambiente de aprendizagem engajador para os estudantes [Acharya and Gayana 2021, Silva et al. 2019].

Diante deste cenário, as Metodologias Ativas (MAs) vêm ganhando destaque entre os docentes [Ribeiro et al. 2021]. Segundo Diesel et al. (2017), as MAs possibilitam uma mudança no paradigma de aprendizagem, onde o estudante sai do papel de agente passivo (apenas escuta e recebe o conteúdo que é transmitido pelo docente) e passa para o papel de agente ativo da aprendizagem, tornando-se o principal responsável por sua aprendizagem. As MAs são estratégias de ensino centradas na participação efetiva dos estudantes e, por conta disso, auxiliam na construção do processo de aprendizagem de uma maneira flexível, interligada e híbrida [Bacich and Moran 2018]. Sob esta perspectiva, é importante refletir sobre à adoção de MAs durante o ensino de programação frente às estratégias de ensino tradicionais que continuam centradas no docente.

Nesse sentido, este artigo tem por objetivo sumarizar as principais MAs adotadas pelos docentes durante o ensino de programação de computadores em cursos de graduação no Brasil. Para isso, foi conduzido um Mapeamento Sistemático da Literatura (MSL) nos principais eventos e periódicos relacionados à Informática na Educação e Educação em Computação no Brasil, entre os anos de 2010 e 2021. Os resultados alcançados mostram um panorama sobre a aplicação das MAs no ensino de programação e as percepções dos estudantes, com relação às MAs, enquanto aprendem conteúdos relacionados à programação de computadores.

\section{Método de Pesquisa}

Um MSL [Kitchenham and Charters 2007] foi realizado para identificar o panorama sobre as MAs adotadas para o ensino de programação de computadores na educação superior no Brasil. Os procedimentos utilizados serão detalhados nas subseções a seguir.

\subsection{Questões de Pesquisa}

Para guiar este trabalho definiu-se as seguintes Questões de Pesquisa (QP):

- QP1: Quais as metodologias ativas que são comumente adotadas por docentes durante o ensino e aprendizagem de programação de computadores?

- QP2: Como os estudantes percebem as metodologias ativas durante a aprendizagem de programação?

\subsection{Estratégia de Busca}

Este MSL se propõe a investigar as MAs adotadas nos cursos de graduação do Brasil. Esta pesquisa foi realizada manualmente nos seguintes eventos e revistas científicas de Educação em Computação e Informática na Educação no Brasil: Simpósio Brasileiro de Informática na Educação (SBIE), Workshop de Informática na Escola (WIE), Workshop de Educação em Computação (WEI), Simpósio Brasileiro de Jogos e Entretenimento Digital (SBGames), Congresso Internacional de Informática Educativa (TISE), Revista Novas Tecnologias na Educação (RENOTE) e Revista Brasileira de Informática na Educação (RBIE). Considerando que a Sociedade Brasileira de Computação vem trabalhando, desde 2010, na reformulação do Currículo de Referência dos cursos de 
Computação [Castro and Siqueira 2019], neste trabalho considerou-se o período de 2010 até 2021, para conhecer o cenário nacional em relação à utilização das MAs na última década. Este MSL foi conduzido até maio de 2021.

\subsection{Seleção das publicações}

Para a seleção das publicações identificadas, os seguintes Critérios de Inclusão (CI) foram definidos: CI1, devem ser selecionadas publicações que apresentam MAs e/ou as percepções dos docentes/estudantes sobre as MAs adotadas durante o ensino de programação; e CI2, devem ser selecionadas publicações que apresentam estudos experimentais sobre o uso das MAs durante o ensino de programação. Também foram definidos os Critérios de Exclusão (CE): CE1, publicações não disponíveis para a leitura e coleta dos dados (publicações pagas, por exemplo); CE2, publicações que não atendam os critérios de inclusão; CE3, publicações que não estejam nos idiomas Português ou Inglês; CE4, publicações duplicadas.

Na primeira etapa do processo de seleção ( $\mathbf{1}^{\mathbf{0}}$ Filtro $)$, realizou-se a leitura do título, palavras-chave e resumo de cada publicação. Neste momento, foram selecionadas as publicações que atendessem pelo menos um dos critérios de inclusão. Em caso de dúvida, a publicação era incluída para uma análise posterior. Na segunda etapa ( $2^{\mathbf{o}}$ Filtro), realizou-se a leitura completa das publicações selecionadas no $1^{\circ}$ Filtro utilizando os critérios de inclusão e exclusão para decidir se publicação seria selecionada ou não.

\subsection{Extração dos dados}

Nesta fase, foram extraídos os dados categorizados da seguinte forma: informações gerais (título, autores, ano, tipo e o local de publicação), metodologia (tipos de MAs identificadas nas publicações), trechos das publicações que apresentavam às percepções dos estudantes sobre as MAs, nome das disciplinas, linguagens de programação mencionadas e informações sobre as ferramentas utilizadas em conjunto com as MAs.

\section{Resultados Obtidos}

A Tabela 1 apresenta a quantidade de publicações retornadas por evento e revista (segunda coluna), bem como a quantidade de artigos selecionados no $1^{\circ}$ e no $2^{\circ}$ Filtro. Um total de 21 publicações foram selecionadas com base nos critérios descritos na Subseção 2.3.

Tabela 1. Quantitativo das publicações retornadas.

\begin{tabular}{c|c|c|c} 
Bibliotecas Digitais & Publicações & $1^{\circ}$ Filtro & $2^{\circ}$ Filtro \\
\hline SBGames & 315 & 16 & 7 \\
\hline SBIE & 305 & 14 & 4 \\
\hline RENOTE & 265 & 5 & 2 \\
\hline RBIE & 336 & 14 & 1 \\
\hline TISE & 21 & 2 & 1 \\
\hline WEI & 88 & 19 & 4 \\
\hline WIE & 177 & 12 & 2 \\
\hline Total & $\mathbf{7 5 3}$ & $\mathbf{2 2 0}$ & $\mathbf{2 1}$ \\
\hline
\end{tabular}

Os resultados mostram que houve um aumento na quantidade de publicações em 2018 e 2019 (Figura 1). No período de 2011 a 2017 observa-se uma oscilação no quantitativo de publicações (entre uma e três publicações por ano). Nos anos de 2012 e 2015 não foram identificadas publicações no contexto deste MSL. Percebe-se que há 
uma quantidade significativa de publicações sobre o uso de MAs para a aprendizagem de programação. Logo, acredita-se que a comunidade está constantemente pesquisando e publicando sobre a adoção de MAs para apoiar a prática docente.

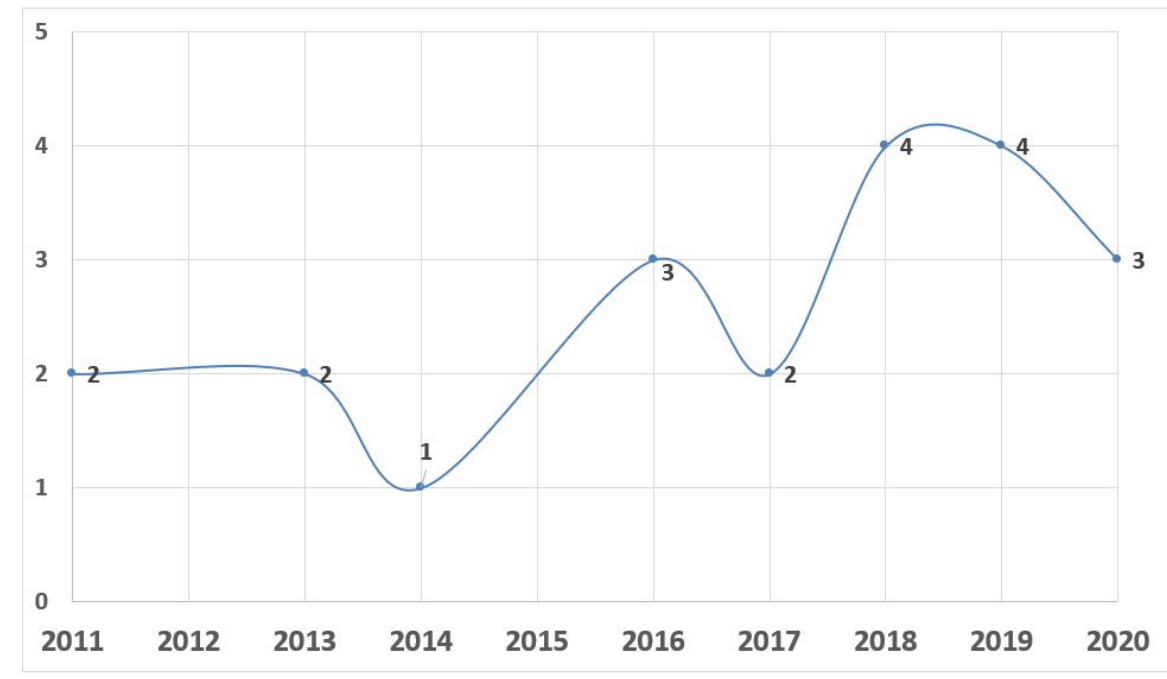

Figura 1. Tendência de publicação por ano.

\subsection{QP1 - Quais as MAs que são comumente adotadas pelos docentes durante o ensino e aprendizagem de programação de computadores?}

Em relação a QP1, a Figura 2 apresenta uma visão geral das MAs identificadas no âmbito deste MSL. Mais detalhes sobre os resultados das MAs podem ser encontradas no relatório técnico (https://figshare.com/s/21338d322bcfed888be5).

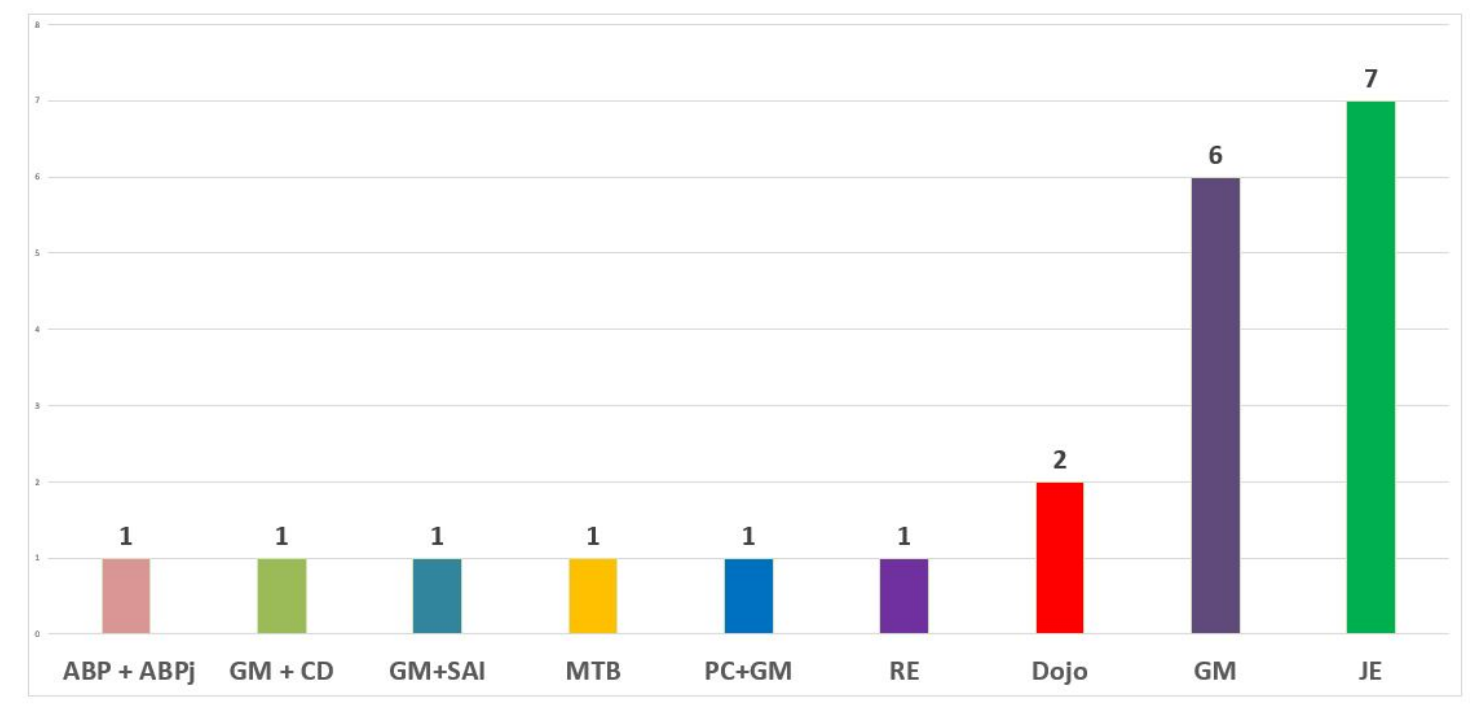

Figura 2. Tipos de Metodologias ativas identificadas nas publicações.

Ao todo, foram identificadas dez (10) tipos de MAs adotadas pelos docentes, são elas: Aprendizagem Baseada em Problemas (ABP); Aprendizagem Baseada em Projetos (ABPj); Coding Dojo (Dojo); Computação Desplugada (CD); Sala de Aula Inve- 
tida (SAI); Gamificação (GM); Jogos Educacionais (JE), Método Baseado em Tutoriais (MBT), Programação Competitiva (PC) e Robótica Educacional (RE).

Na Figura 2 percebe-se que Jogos Educacionais é a metodologia com maior quantidade de publicações disponíveis (7) e, consequentemente, a mais adotada pelos docentes. Destaca-se que, no contexto dos JEs, existe uma associação automática dessa MA com jogos digitais. Stephan et al. (2020) apresentam o GameProgJP, uma abordagem que visa apoiar o ensino de programação empregando como recurso pedagógico o desenvolvimento de jogos. O jogo é divido em quatro partes, sendo que cada parte abordava determinado conteúdo da disciplina: $1^{\text {a }}$ parte, declaração de variáveis e uso de funções; $2^{\mathrm{a}}$ parte, estruturas condicionais; $3^{\mathrm{a}}$ parte, estruturas de repetição, vetores e strings; $4^{\mathrm{a}}$ parte, matrizes e estruturas de dados heterogêneas. Ao final são geradas quatro versões do jogo. As mecânicas do jogo são implementadas de acordo com o conteúdo ensinado ao longo da disciplina [Stephan et al. 2020]. Como resultados, os autores notaram que a abordagem motivou e aumentou o interesse dos estudantes por programação.

No que diz respeito à Gamificação (GM), essa MA vem sendo utilizada pelos docentes pois torna a prática das disciplinas de programação uma atividade leve, divertida e descontraída para os estudantes [Casarotto et al. 2018]. Para auxiliar neste processo, os docentes têm adotado ferramentas e atividades gamificadas com foco na resolução de problemas, como o Kahoot e Socrative [Nagai et al. 2016], o Portal URI Online Judge [Brito et al. 2019] e o UVa Online Judge [Melo et al. 2016]. Outro ponto a ser destacado é que a GM normalmente vem sendo adotada em conjunto com outras MAs. Costa et al. (2017) relatam uma experiência sobre uso de elementos de gamificação combinados com as metodologia SAI para ensinar os conteúdos de Programação Orientada à Objetos. Como resultados, os autores comentam que à adoção combinada destas MAs permitiu a melhoria do rendimento acadêmico e do engajamento dos estudantes nas aulas.

Foram identificadas também as disciplinas de programação em que as MAs foram adotadas, são elas: Algoritmos (AL), Algoritmos II (ALII), Estruturas de Dados (ED), Introdução à Programação (IP), Laboratório de Programação (LProg), Linguagem de Programação (LP), Lógica de Programação (LogProg), Programação Orientada à Objetos (POO), Programação (Prog), Programação Web (ProgWeb) e Teoria dos Gráfos (TG). Observou-se que a linguagem de programação $\mathrm{C}$ foi a mais utilizada pelos docentes para ensinar programação, seguida pela linguagem Java e Python. A Tabela 2 apresenta um resumo das MAs encontradas, as disciplinas ao qual foram adotadas e, quando houver, as ferramentas utilizadas pelos docentes para apoiar na adoção das MAs. ${ }^{1}$

Tabela 2. MAs utilizadas por disciplina e ferramentas utilizadas.

\begin{tabular}{ll} 
MAs & Nome das Disciplinas e Ferramentas Adotadas \\
\hline JE & $\begin{array}{l}\text { LogProg e AL (Jogo Logirunner), IP (Jogo Klouro), POO (Jogo Gaia ABstração Game, POOkemon), Prog } \\
\text { (Jogos Bullfrogs, Metrocity, Carcassonne e o jogo Um império em oito minutos) e LProg (GameProgJF) }\end{array}$ \\
\hline GM & $\begin{array}{l}\text { POO (cod[edu]), Prog (Kahoot, code.Org, Socrative), IP (Canvas e Huxley), ALII (on-line UVa Online } \\
\text { Judge e o Jogo Game of Code (Goc)) }\end{array}$ \\
\hline Dojo & AL, IP, LP (IDE DevC++) \\
\hline GM + SAI & POO (Moodle) \\
\hline MBT & Prog (FlashPunk) \\
\hline PC + GM & AL, ED (URI online Judge) \\
\hline RE & Prog (Code Blocks e Arduíno) \\
\hline
\end{tabular}

\footnotetext{
${ }^{1}$ Para mais detalhes, ver o relatório técnico deste MSL (https://figshare.com/s/21338d322bcfed888be5).
} 
Em se tratando de ensino de programação, o uso de ferramentas para a execução de tarefas é indispensável. Diante disso, é fato que utilizar as MAs com o suporte ferramental de ferramentas (UVa Online Judge, Kahoot, code.Org e Socrative, por exemplo) contribui para atrair a atenção do estudante em diversas ocasiões nas aulas. Além disso, apoia no engajamento e motivação dos estudantes, em especial nos momentos iniciais do ensino, visto que é imprescindível para o processo de ensino e aprendizagem. Desta forma, notase que a utilização de ferramentas computacionais, aliadas as MAs, contribui para que a construção e compartilhamento do conhecimento dos estudantes. O uso de ferramentas poderá ser uma contribuição pedagógica quando na transposição didática no ensino frente ao perfil dos estudantes na contemporaneidade. Porém, ressalta-se que nem sempre o fato de utilizar alguma ferramenta garante que os objetivos de aprendizagem sejam alcançados. Isso dependerá, muitas vezes, do perfil do estudante, do contexto, da disciplina e do conteúdo em que a ferramenta foi aplicada [Blatt et al. 2017, Silva et al. 2019].

\subsection{QP2 - Como os estudantes percebem as MAs durante a aprendizagem de programação?}

Para responder a QP2, realizou-se uma análise qualitativa das percepções dos estudantes coletadas a partir de cada publicação aceita, em relação as MAs adotadas. O objetivo principal desta análise foi identificar os pontos positivos e negativos relatados na literatura, sob a ótica dos estudantes, em relação às MAs. Para realizar a análise qualitativa, foi criada uma lista com todas as percepções identificadas. Cada uma das percepções foi analisada e, a partir disso, criaram-se códigos. Em seguida, estes códigos foram analisados e agrupados de acordo com as suas características, formando conceitos relevantes e que são representados neste trabalho por meio de Categorias e Subcategorias. Ressalta-se que um pesquisador-autor realizou a análise. Em seguida, ela foi revisada e discutida com outro pesquisador-autor, que possui mais de seis anos de experiência em análise qualitativa. A Tabela 3 mostra as percepções dos estudantes agrupadas de acordo com as Categorias identificadas: Engajamento, Desempenho, Interação e Colaboração, e Motivação.

Tabela 3. Percepções dos Estudantes em relação às MAs.

\begin{tabular}{|c|c|c|c|c|c|c|c|c|c|c|c|c|}
\hline Cat. & Subcategorias & $\mathrm{ABP}$ & $\mathrm{ABPj}$ & $\mathrm{CD}$ & Dojo & $\mathrm{PC}$ & GM & SAI & $\mathrm{JE}$ & MBT & RE & \#artigos \\
\hline \multirow{3}{*}{ 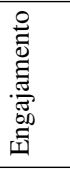 } & $\begin{array}{l}\text { (+) Engajados para aprender os conteúdos ensi- } \\
\text { nados }\end{array}$ & $\mathrm{X}$ & & $\mathrm{X}$ & & & $\mathrm{X}$ & & $\mathrm{X}$ & & & 06 \\
\hline & (+) Engajados devido ao trabalho em equipe & $\mathrm{X}$ & & & $\mathrm{X}$ & $\mathrm{X}$ & & & & & & 01 \\
\hline & $\begin{array}{l}\text { (+) Engajados devido aos momentos de dis- } \\
\text { cussão e de tirar dúvidas durante a aula }\end{array}$ & & & & & & $\mathrm{X}$ & & $\mathrm{X}$ & & & 02 \\
\hline \multirow{4}{*}{ 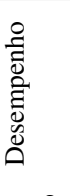 } & (+) Melhoria no desempenho na disciplina & & & $\mathrm{X}$ & & $\mathrm{X}$ & $\mathrm{X}$ & $\mathrm{X}$ & & & & 03 \\
\hline & $\begin{array}{l}\text { (+) Melhoria do desenvolvimento de com- } \\
\text { petências profissionais }\end{array}$ & $\mathrm{X}$ & $\mathrm{X}$ & & $\mathrm{X}$ & $\mathrm{X}$ & & & & & & 02 \\
\hline & (+) Melhoria nas habilidades de programação & & & & & $\mathrm{X}$ & $\mathrm{X}$ & $\mathrm{X}$ & & & & 01 \\
\hline & $\begin{array}{l}\text { (+) Contribuiu para o entendimento dos concei- } \\
\text { tos ensinados }\end{array}$ & & & & $\mathrm{X}$ & & & & $\mathrm{X}$ & & & 04 \\
\hline \multirow{2}{*}{ 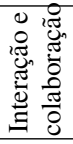 } & (+) Melhoria da participação em sala de aula & $\mathrm{X}$ & & & $\mathrm{X}$ & $\mathrm{X}$ & $\mathrm{X}$ & & & & & 03 \\
\hline & $\begin{array}{l}\text { (+) Incentivou a colaboração e interação en- } \\
\text { tre estudantes e estudantes e docentes para } \\
\text { resolução dos exercícios }\end{array}$ & $\mathrm{X}$ & & & $\mathrm{X}$ & $\mathrm{X}$ & $\mathrm{X}$ & & & & & 02 \\
\hline \multirow{4}{*}{ 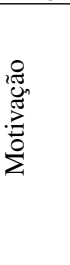 } & $\begin{array}{l}\text { (+) Motivou os estudantes aprenderam de forma } \\
\text { divertida a disciplina }\end{array}$ & & & & $\mathrm{X}$ & $\mathrm{X}$ & $\mathrm{X}$ & & $\mathrm{X}$ & $\mathrm{X}$ & $\mathrm{X}$ & 04 \\
\hline & $\begin{array}{l}\text { (+) Motivou os estudantes a continuarem fre- } \\
\text { quentando a disciplina }\end{array}$ & & & & & & $\mathrm{X}$ & & & $\mathrm{X}$ & & 01 \\
\hline & $\begin{array}{l}\text { (+) Motivou os estudantes a se sentiram envol- } \\
\text { vidos no aprendizado }\end{array}$ & & & & $\mathrm{X}$ & $\mathrm{X}$ & $\mathrm{X}$ & & $\mathrm{X}$ & & $\mathrm{X}$ & 04 \\
\hline & $\begin{array}{l}\text { (+) Possibilitou os estudantes buscarem diferen- } \\
\text { tes formas de resolver os problemas }\end{array}$ & & & & $X$ & & $\mathrm{X}$ & & $\mathrm{X}$ & & & 03 \\
\hline
\end{tabular}


Para a categoria "Engajamento" foram associadas três subcategorias que retratam os motivos pelos quais os estudantes se sentiram mais engajados para aprender programação com aquelas MAs. A percepção em relação ao engajamento foi identificada quando os docentes empregaram as seguintes MAs: ABP, CD, Dojo, GM e JE. Observa-se que estas MAs contribuem para o despertar dos estudantes para uma postura ativa, criativa e colaborativa, visto que se mostram engajados no trabalho em equipe e para discutir as questões durante a aula, buscando tirar dúvidas.

Na categoria "Desempenho", as seguintes MAs se destacaram: ABP, ABPj, CD, Dojo, PC, GM, SAI e JE. Nota-se que todas elas estão relacionadas à melhoria do desempenho dos estudantes e algumas mais especificamente ao desenvolvimento de competências profissionais, como comunicação mais ampla, trabalho em equipe e autodidatismo. Além disso, nota-se uma discussão sobre a melhoria das habilidades de programação, como a capacidade para resolução de problemas, entendimento do funcionamento básico da linguagem de programação e a capacidade de leitura de código [Nagai et al. 2016], além de contribuir para o entendimento dos conceitos ensinados. Deste modo, os estudantes conseguem desenvolver habilidades relacionadas aos conhecimentos e práticas da programação, construindo competências profissionais.

Partindo do pressuposto que a construção do conhecimento perpassa pela troca de experiências e compartilhamento do conhecimento construído, observou-se que as MAs ABP, Dojo, PC e GM foram as que mais contribuíram para o despertar da "Interação e Colaboração" entre os estudantes e entre os estudantes e docentes. Isto ocorre, pois estas MAs permitem a melhoria da participação em sala de aula e troca de conhecimentos por meio da interação nas discussões. Por fim, observa-se que a categoria "Motivação" esteve associada a forma divertida adotada para ensinar o conteúdo (MAs CD, Dojo, PC, GM, JE, MBT e RE). A motivação dos estudantes refletiu numa melhoria na frequência com que os estudantes participavam das aulas, uma vez que eram desafiados a buscarem formas inovadoras de resolver os problemas, dentro e fora da sala de aula.

Em relação as percepções negativas das MAS, tais percepções estavam relacionadas a baixa compreensão dos estudantes sobre os conteúdos das disciplinas e não sobre às MAs em si, tais como [Silva et al. 2018, Moreira and Monteiro 2018]: dificuldade de compreender o funcionamento das estruturas de controle, dificuldade de criar algoritmos que resolvam problemas concretos; dificuldade em aprender a pensar algoritmicamente, dificuldades de se posicionar em frente aos colegas e docentes para expor o seu trabalho e responder a questionamentos. Também houveram aspectos negativos relacionados a programação e ao funcionamento do computador, e dificuldades sobre questões técnicas, por exemplo, a manipulação de arquivos em Java [Cera et al. 2012], falta de habilidades necessárias, como a resolução de problemas [Nagai et al. 2016] ou mesmo a falta de motivação dos estudantes e sua dificuldade para manter um ritmo de estudos contínuo [Raposo and Dantas 2016]. Acredita-se que as percepções identificadas e mapeadas podem subsidiar a construção de conhecimentos para a escolha das MAs a serem utilizadas, frente ao objetivo de aprendizagem a ser alcançado. Além disso, o interesse em conhecer a percepção dos estudantes é importante, pois possibilita, por exemplo, saber se alguns estudantes precisam de ajuda adicional para compreender os conteúdos debatidos em sala de aula ou mesmo para promover (novos) meios para ajudar nas dificuldades apresentadas. 


\section{Discussão dos Resultados}

Os resultados apresentados neste trabalho mostram que há uma preocupação relacionada à adoção / aplicação de diferentes MAs durante o ensino de programação, haja vista que vem sendo exigido cada vez mais do corpo docente a preparação de aulas dinâmicas e o domínio de como utilizar as diferentes MAs para motivar e engajar os estudantes. Percebe-se também um número crescente de MAs que vêm ganhando destaque, no sentido de chamar a atenção dos estudantes e mudando a forma tradicional de aprendizagem de programação [Castro and Siqueira 2019]. Frente a isso, as pesquisas identificadas neste MSL mostram a importância de se explorar esse tema e a necessidade de desenvolver mais estudos para um melhor entendimento de como e quando utilizar MAs no ensino da programação nos cursos da área da Computação e frente as diferentes necessidades dos envolvidos no processo de ensino e aprendizagem no cenário educacional.

Além disso, esse panorama demonstra que foram encontrados mais benefícios do que dificuldades, em especial na perspectiva do estudante, em relação a adoção das MAs nas aulas de programação. Portanto, conclui-se que a adoção de MAs, de maneira geral, vem produzido resultados de aprendizagem positivos para os estudantes, uma vez que de certa forma as MAs fazem com que os estudantes rompam com sua passividade em sala de aula [Mulcahy 2002]. Desta forma, é necessário apoiar e capacitar o corpo docente para que possam atender às necessidades do ensino [Borges and Alencar 2014].

Ao observar a literatura, identificou-se os trabalhos de Borges et al. (2018) e Medeiros et al. (2018) . Contudo, estes trabalhos olham para a literatura sob a ótica de estratégias de ensino (tradicionais ou não) que podem ser adotadas durante o ensino de programação, diferentemente deste MSL, que foca apenas nas MAs. O trabalho de Berssanette e Francisco (2021) apresenta os resultados de MSL em que foram identificas estratégias pedagógicas adotadas no ensino de programação, similarmente ao conduzido neste MSL. Constatou-se que apesar do estudo ter sido executado apenas no contexto brasileiro e com foco no ensino superior, os resultados encontrados neste MSL estão alinhados com os resultados de Berssanette e Francisco (2021).

\section{Considerações Finais}

O foco desta pesquisa é sumarizar as principais MAs utilizadas no ensino de programação em cursos superiores no Brasil, além de relatar as percepções dos estudantes sobre o processo de aprendizagem com estas MAs. Para isso, foi conduzido um MSL nos principais eventos e revistas científicos do Brasil. A partir dos resultados alcançados, foram identificadas que dez metodologias ativas são comumente adotadas pelos docentes.

As MAs que mais se destacaram foram JE e GM, estas MAs podem fornecer um feedback rápido, já que muitas vezes são apoiadas por ferramentas tecnológicas e que visam facilitar o processo de aprendizagem de conceitos associadas à prática das teorias aprendidas e a colaboração entre os estudantes. Também, tais ferramentas fornecem meios para que as práticas para a aprendizagem possam ser realizadas em equipe, o que desperta a curiosidade, motiva e engaja os estudantes a aprender, a compartilhar e buscar ativamente a construções de seus novos conhecimentos, praticando os conceitos com a utilização e o suporte de tecnologias físicas ou digitais.

Como contribuições, este trabalho apresenta diferentes MAs para suporte ao ensino e a aprendizagem ativa da programação. Isto é necessário pois, muitas vezes, os 
docentes não sabem qual MA adotar em sala de aula frente aos diferentes assuntos e disciplinas que precisa ministrar ao longo do semestre. Como trabalhos futuros pretende-se desenvolver um repositório colaborativo aberto em que os docentes possam identificar, selecionar, adotar, discutir, comentar, avaliar e possivelmente colaborar com (novas ou não) MAs utilizadas durante o ensino de programação.

\section{Referências}

Acharya, S. and Gayana, M. (2021). Enhanced learning and improved productivity of students' using project based learning approaches for programming courses. Journal of Engineering Education Transformations, 34:524-530.

Bacich, L. and Moran, J. (2018). Metodologias ativas para uma educação inovadora: uma abordagem teórico-prática. Penso Editora.

Berssanette, J. and de Francisco, A. (2021). Active learning in the context of the teaching/learning of computer programming: A systematic review. Journal of Information Technology Education: Research, 20(1):201-220.

Blatt, L., Becker, V., and Ferreira, A. (2017). Mapeamento sistemático sobre metodologias e ferramentas de apoio para o ensino de programação. In Anais do Workshop de Informática na Escola, volume 23, page 815.

Borges, R., Oliveira, P. R. F., Lima, R. d. R., and De Lima, R. (2018). A systematic review of literature on methodologies, practices, and tools for programming teaching. IEEE Latin America Transactions, 16(5):1468-1475.

Borges, T. S. and Alencar, G. (2014). Metodologias ativas na promoção da formação crítica do estudante: o uso das metodologias ativas como recurso didático na formação crítica do estudante do ensino superior. Cairu em revista, 3(4):119-143.

Brito, P., Fortes, R., Faria, F., Lopes, R. A., Santos, V., and Magalhães, F. (2019). Programação competitiva como ferramenta de apoio ao ensino de algoritmos e estrutura de dados para alunos de ciência da computação. In Simpósio Brasileiro de Informática na Educação (SBIE), volume 30, page 359.

Casarotto, R. I., Bernardi, G., Cordenonsi, A. Z., and Medina, R. D. (2018). Logirunner: um jogo de tabuleiro como ferramenta para o auxílio do ensino e aprendizagem de algoritmos e lógica de programação. RENOTE, 16(1).

Castro, R. M. and Siqueira, S. (2019). Técnicas alternativas de ensino (aprendizagem ativa) para disciplinas da computação: Um mapeamento sistemático no contexto brasil. In Anais do Workshop de Informática na Escola, volume 25, pages 1409-1413.

Cera, M. C., Dal Forno, M. H., and Vieira, V. G. (2012). Uma proposta para o ensino de engenharia de software a partir da resolução de problemas. Revista Brasileira de Informática na Educação, 20(03):116.

Costa, A. F. F., de MELO, A. F. M. F., MOREIRA, G. G., CARVALHO, M. d. A., and LIMA, M. V. d. A. (2017). Aplicação de sala invertida e elementos de gamificação para melhoria do ensino-aprendizagem em programação orientada a objetos.

Diesel, A., Baldez, A. L. S., and Martins, S. N. (2017). Os princípios das metodologias ativas de ensino: uma abordagem teórica. Revista Thema, 14(1):268-288. 
Kitchenham, B. and Charters, S. (2007). Guidelines for performing systematic literature reviews in software engineering. Technical report, Keele University and Durham University Joint Report.

Luxton-Reilly, A., Albluwi, I., Becker, B. A., Giannakos, M., Kumar, A. N., Ott, L., Paterson, J., Scott, M. J., Sheard, J., and Szabo, C. (2018). Introductory programming: a systematic literature review. In Proceedings of the 23rd Annual ACM Conference on Innovation and Technology in Computer Science Education, pages 55-106.

Medeiros, R. P., Ramalho, G. L., and Falcão, T. P. (2018). A systematic literature review on teaching and learning introductory programming in higher education. IEEE Transactions on Education, 62(2):77-90.

Melo, S., Oliveira, R., and Soares Neto, C. d. S. (2016). Game of code: aplicando gamificação em disciplinas de programação. In XV Simposio Brasileiro de Jogos e Entretenimento Digital (SBgames 2016).

Moreira, J. A. and Monteiro, W. M. (2018). O uso da computação desplugada em um contexto de gamificação para o ensino de estrutura de dados. RENOTE, 16(2):546555 .

Mulcahy, D. (2002). Team-based learning: A transformative use of small groups. Greenwood publishing group.

Nagai, W., Izeki, C., and Dias, R. (2016). Experiência no uso de ferramentas online gamificadas na introdução à programação de computadores. In Anais do Workshop de Informática na Escola, volume 22, page 301.

Raj, A. G. S., Patel, J., and Halverson, R. (2018). Is more active always better for teaching introductory programming? In 2018 International Conference on Learning and Teaching in Computing and Engineering (LaTICE), pages 103-109. IEEE.

Raposo, E. H. S. and Dantas, V. (2016). O desafio da serpente-usando gamification para motivar alunos em uma disciplina introdutória de programação. In Simpósio Brasileiro de Informática na Educação (SBIE), volume 27, page 577.

Ribeiro, I. C., Silva, W., and Feitosa, E. L. (2021). Repositório colaborativo para apoiar a adoção de metodologias ativas no ensino de programação. In Anais Estendidos do Simpósio Brasileiro de Educação em Computação, pages 56-57. SBC.

Silva, T. S. C., de Melo, J. C. B., and Tedesco, P. C. d. A. R. (2018). Um modelo para promover o engajamento estudantil no aprendizado de programação utilizando gamification. Revista Brasileira de Informática na Educação, 26(03):120.

Silva, W., Steinmacher, I., and Conte, T. (2019). Students' and instructors' perceptions of five different active learning strategies used to teach software modeling. IEEE Access, 7:184063-184077.

Stephan, J., Oliveira, A., and Renhe, M. C. (2020). O uso de jogos para apoiar o ensino e aprendizagem de programação. In Anais do XXXI Simpósio Brasileiro de Informática na Educação, pages 381-390. SBC. 